\title{
Computer Modeling for Fire and Smoke Dynamics in Enclosures: A Help or a Burden?
}

\author{
BART MERCI \\ Department of Flow, Heat and Combustion Mechanics \\ Ghent University - UGent \\ EA03, Sint-Pietersnieuwstraat 41 \\ B-9000 Ghent, Belgium \\ e-mail: Bart.Merci@UGent.be
}

\begin{abstract}
Fire simulations are of unique value in different respects: for fire safety system design calculations, for improving our understanding (theory and model development and validation) and for fire forecasting. In this paper the effective use of computing power for the further development of fire safety science is discussed, considering only gas phase phenomena in fire and smoke dynamics in enclosures. Arguably, much effort needs to be devoted to multi-phase phenomena (pyrolysis modeling, the effect of water or other suppressants, etc.), but this is not discussed in the paper at hand.
\end{abstract}

A common feature to all types of simulations is that the Required Computing Resources (RCR), determined by the envisaged accuracy and the complexity of the problem to be tackled, must be less than the Available Computing Resources (ACR). Accuracy, reliability and dimensionality of the models used, must therefore be related to the problem tackled. In order to make progress, bench-marking studies, as a joint effort made by modelers and experimentalists, with transparent communication, are argued to be a good approach for systematic progress in the development of, and confidence in, models.

Using Computational Fluid Dynamics (CFD) can be very valuable for the development of theory and the study of detailed fluid mechanics phenomena, but several aspects are important to guarantee the quality of the results. Some ideas will be formulated on how to investigate requirements on the computational mesh. Turbulence - chemistry interaction (TCI) and turbulence - radiation interaction (TRI) are also discussed briefly. Yet, it is argued that a major source of uncertainty in computer simulations stems from (and will continue to stem from) the characterization of the ever changing and developing materials (i.e. the fuel), as well as from geometry dependent features (including ventilation and heat transfer). This affects the combustion and soot formation, and therefore the fire and smoke dynamics. Therefore, a user-defined fire will remain indispensible in the foreseeable future when using computer modeling for the sake of design of fire safety systems. Once this fire has been defined, CFD is best suited in regions where detail is required or complex flow patterns establish, while other forms of modeling can be considered in other regions.

For real-time and forecasting applications, it is argued that sensor-assisted numerical simulations are very promising and their use is expected to become widespread in the coming decades. With increasing computing power, the use of CFD will become more feasible in this context, but for the time being zone model calculations (perhaps combined with CFD in regions where more detail is required) seem better suited to that purpose.

KEYWORDS: modeling, compartment fires, fluid dynamics, CFD, fire dynamics, smoke

\section{INTRODUCTION}

The ever increasing computing power, stemming from both hardware and software development, calls for a discussion on the use of computer simulations of fire and smoke dynamics in enclosures. Indeed, this technological evolution opens up tremendous possibilities for the further development of fire safety science. The question must, however, be addressed as to how this power can be used in an effective manner. This implies sufficient investment in resources, but also a high level of competence of the people involved. Indeed, when not used properly, computer simulations might lead to erroneous conclusions. In such circumstances, they are a burden, rather than a help, in the process of fire safety science development. To a certain extent, this also relates to the simulation tool applied: a more 'fail-safe' tool can compensate a certain 'lack of competence' of the user. 
The reader may notice that the title refers to 'modeling', whereas the text so far mentions 'simulations'. Because all practically relevant fire and smoke simulations require modeling, both terms are used without distinction in the present paper and, e.g., numerical accuracy will also be labeled simply as 'accuracy', in the context of modeling reality.

This paper is not meant to be a review that describes the state-of-the-art from the perspective of the past. Rather, the author's intention is to formulate suggestions and opinions, based on the state-of-the-art and the ongoing evolutions. Obviously, the reader can agree with these opinions or not. Yet, it is the author's ambition to explain on what facts the opinions and suggestions rely. Reference will be made to a couple of 'examples', but the list of references is by no means intended to cover the state-of-the-art completely. In other words, many valuable papers and books are not referred to, even though they would deserve to be cited if this paper were to be a review paper. In the same sense, no explicit reference is made to existing software packages, since the statements made are not related to specific codes.

The paper also only focuses on gas phase phenomena. This by no means implies that modeling efforts must focus on the gas phase primarily in the coming decade(s). The solid and/or liquid phase, e.g. in the context of pyrolysis or fire suppression, deserve much attention in the fire safety science community. However, the points made below are believed to be relevant in the framework of fire safety systems design (where e.g. pyrolysis processes are typically not modeled explicitly). They are also believed to be relevant for the development of fire safety science, since the main points made also apply to making progress in the modeling of phenomena related to the condensed phase. However, there is no room to discuss each and every single feature within one paper. For these reasons, the choice has been made to primarily discuss gas phase phenomena.

The structure of the paper is as follows. The notions 'Available Computing Resources' (ACR) and 'Required Computing Resources' (RCR) are introduced first. The latter relates to accuracy (e.g. the computational mesh) requirements and the necessary dimensionality of the models used, as well as to the complexity of the problem to be tackled. Within the context of the present paper, fire modeling primarily involves turbulent reactive and non-reactive conditions in gas phase simulations. Given the nature of the problem, many phenomena cannot be fully resolved (as ACR $<<\mathrm{RCR}$ then), so 'modeling' is required. The approach to be adopted depends on the objective (design of fire safety systems, development and validation of models and theories, or fire forecasting).

Next, the use of computer simulations as a tool in the context of design of fire safety systems is discussed. Starting from a state-of-the-art example from the combustion community, ACR-RCR limitations associated to design related simulations are discussed. It is also argued that a major source of uncertainty in computer simulations stems from (and will continue to stem from) the characterization of the ever changing and developing materials, constituting the fuel, as well as from geometry dependent features (including ventilation and heat transfer). This affects the combustion and soot formation, and therefore the fire and smoke dynamics. Therefore, a user-defined fire will remain indispensible in the foreseeable future when using computer modeling for the sake of design of fire safety systems, effectively circumventing the ACRRCR limitations and the uncertainties mentioned. The section closes with some statements on the state-ofthe-art and some suggestions on how to proceed beyond.

Computer simulations can also be used as support for the development and verification of theories and models. It is argued that experimental findings can be confirmed and perhaps extrapolated from simulation results, or aspects can be studied in detail to an extent beyond experimental capabilities. It is suggested that a strong collaborative joint effort is required from experimental and numerical researchers to make systematic progress in the field of using simulation results in this context. In addition to important ongoing verification and validation $(\mathrm{V} \& \mathrm{~V})$ efforts within the fire community, two enlightening examples from the combustion community are briefly discussed. Some attention is devoted to turbulence-chemistry interaction (TCI) and turbulence-radiation interaction (TRI), but it is argued that the major task for the fire community would be to focus on typical fire related topics (such as: combustion mechanisms at relatively low temperature and in under-ventilated conditions, including soot formation and $\mathrm{CO}$ production and consumption; pyrolysis modeling; interaction with water), and to exploit knowledge from other communities (particularly the combustion community) where possible. Bench-marking studies are argued to be particularly appealing, effectively trying to reduce uncertainties at the level of boundary conditions (particularly at the level of type and amount of fuel), therefore allowing the study of specific modeling 
aspects. The reduced uncertainty at the level of boundary conditions is an essential difference from the design oriented simulations.

The final section focuses on the use of computer calculations in the context of real-time fire evolution and even fire forecasting, which is a third possible objective of simulations. As the calculations need to be faster than real-time, most phenomena need to be modeled. It is argued that the use of sensor-assisted numerical simulations is expected to become widespread in the coming decades, as such circumventing the uncertainties inherent to the boundary conditions of the problem at hand. With increasing computing power, the use of CFD will become more feasible in this context, but for the time being, zone model calculations (perhaps combined with CFD in regions where more detail is required) are argued to be better suited for fire forecasting purposes.

In summary, three types of simulations are discussed: simulations for the design of fire safety systems, for the development and validation of theories and models, and for fire forecasting purposes. The author's opinion on how to proceed from the current state-of-the-art in each type is formulated, providing example and suggestions for possible strategies.

\section{AVAILABLE COMPUTING RESOURCES (ACR) VERSUS REQUIRED COMPUTING RESOURCES (RCR)}

The terminology Available Computing Resources (ACR) and Required Computing Resources (RCR) is introduced first. [If not all available resources are effectively used, ACR could also stand for 'Applied Computing Resources'.] As long as ACR > RCR, and assuming that the simulation tools are used in a technically correct manner (see below), the simulation results can be reliable. Indeed, an illustration is provided below, showing that complex problems can be tackled in an accurate manner with state-of-the-art CFD for turbulent reacting flows. However, the practice in fire safety science and engineering simulations is that ACR can be lower than RCR. This is discussed next.

Figure 1 visualizes the relation between RCR and three aspects of CFD simulations:

- Complexity: this refers to geometrical complexity of the configuration at hand, the number and complexity of scenarios, inclusion of multiple required outputs (temperature, species, etc.), two-phase phenomena, etc.;

- Accuracy: this refers to numerical aspects (computational mesh, numerical schemes, convergence levels, etc.) and to modeling aspects (computational mesh, combustion model, radiation model, soot model, pyrolysis model, etc.);

- Modeling dimensionality: this refers to aspects that are modeled (or not). To give an example for smoke dynamics: from a flow dynamics point of view, hot air resembles smoke. Thus, if the question remains as 'simple' as to determine whether or not smoke is present at a certain moment in time at a certain location in a building, given a fire source and perhaps an active smoke control system, the simulations can be restricted to the flow of 'hot air'. However, if toxicity (even at the level of CO concentrations alone) and optical thickness are an issue, soot modeling and chemistry and species transport come into play as well. In other words: the required 'dimensionality' of modeling strongly depends on the problem to be tackled.

Note that there is no scale on the axes in Fig. 1 and that the lines are merely indicative. The dependency of RCR on complexity, accuracy and modeling dimensionality need not be identical. Also, the lines are sketched as curves with a stronger increase than linear, but in some cases the dependency can be linear. For example, if the increase in complexity merely involves an increase in number of (similar) scenarios, the RCR would increase linearly. If, however, the step is made from the binary question of whether or not smoke is present to the question on optical thickness, the dependence will be stronger than linear. Even discontinuities in the lines are possible. Also note that the three aspects mentioned interact with each other. Indeed, using still the same example of smoke, the question for toxicity not only increases complexity, it also forces the modeling to be more 'multi-dimensional' in that (finite rate) chemistry and species transport must be accounted for. The latter can pose additional requirements on the accuracy level. A similar argument holds for e.g. an activated sprinkler system (multi-phase phenomena). Therefore, the 'combined' line can be quite steep. While it may be impossible to achieve with great accuracy, quantification of the 
position of the ACR and RCR lines is considered an important ambition for the fire safety science community, as explained below.

The horizontal dash-dotted line in Fig. 1 refers to the ACR. Obviously, this line will shift upward in the future thanks to evolution in hardware and software. It can also be shifted upward by investments in hardware and massively parallel computing. Note that the factor 'time' is implicitly present in Fig. 1. At the level of RCR, this is involved in the duration of the scenario that needs to be computed. The longer the scenario, the higher RCR becomes. This increase can be strongly nonlinear or even discontinuous (e.g. if multiple burning objects enter the scenario, or if a sprinkler system is activated, or if the fire evolves from well-ventilated to under-ventilated, etc.). At the level of ACR, time relates to the time taken to perform the calculations. Slower hardware, used over a longer period in time, yields a similar ACR as faster hardware during a shorter period in time.

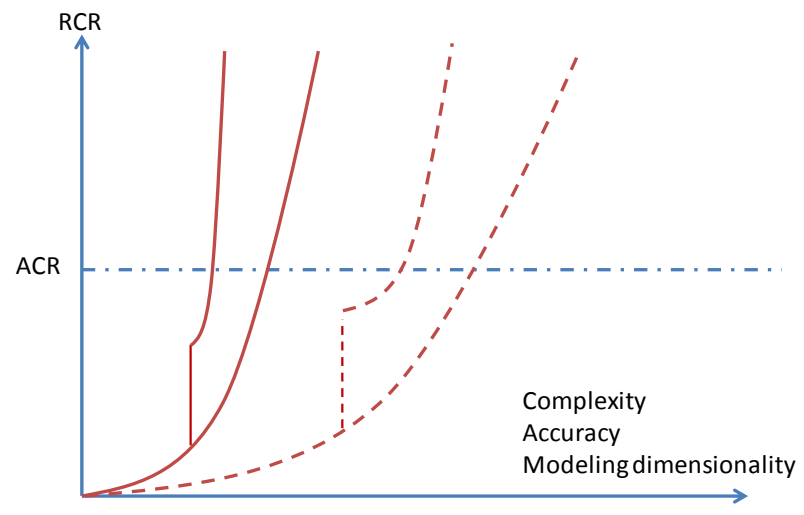

Fig. 1. Dependency of RCR on complexity, accuracy and modeling dimensionality. Lines are merely indicative. Discontinuities in the lines (indicated as sudden jumps in the lines) are possible. Dashed: individual dependency; solid: combined dependency; horizontal dash-dotted line: ACR.

Figure 2 visualizes the information in another manner. For a given amount of ACR, the choice must be made between investing the ACR in increased complexity (e.g. more or longer or more complex scenarios, more complex geometry, more complex design questions (e.g. temperature or species concentration fields)) or in increased modeling dimensionality (e.g. inclusion of soot modeling or more complete combustion or radiation modeling) or increased accuracy (e.g. more accurate numerical scheme or finer computational mesh). Obviously combinations are possible. Again, the lines in Fig. 2 are merely indicative. For example, infinite complexity or accuracy or modeling dimensionality cannot be achieved. Still, it is again important to try and determine whether or not the RCR, related to the problem at hand, exceeds the ACR and if so, what is the effect on the reliability of the simulation results.

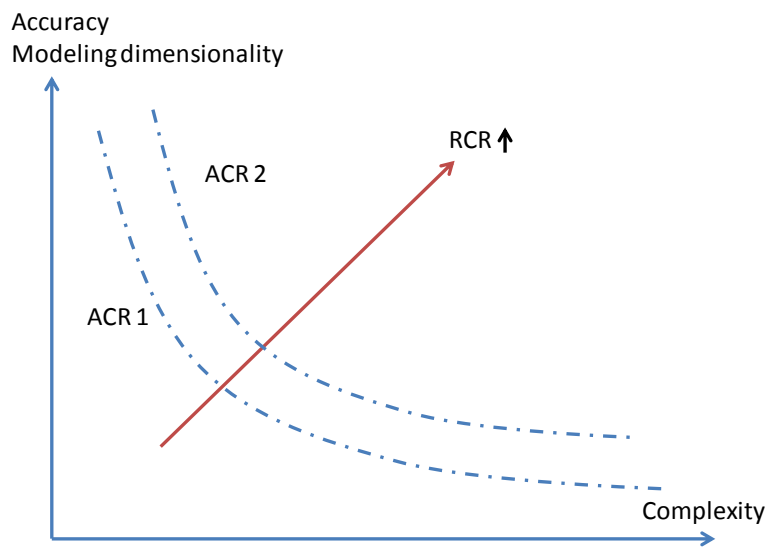

Fig. 2. Trade-off between complexity on the one hand and accuracy and modeling dimensionality on the other. Lines are merely indicative. ACR $2>$ ACR 1. 
To conclude this section, it is noted that the numerical scheme in a CFD code can effectively reduce the RCR, given a certain level of accuracy, complexity and modeling dimensionality. Or, stated in another manner, higher complexity can be pursued with a given amount of ACR, keeping accuracy and modeling dimensionality unaffected, if the numerical solution procedure is faster. However, problems may arise if, for the sake of reduction of RCR, the numerical scheme and/or the computational mesh are chosen such that a hidden price is paid at the level of accuracy or modeling dimensionality. The next section devotes a discussion to the computational mesh.

\section{Requirements for the computational mesh}

As mentioned, Fig. 2 visualizes the trade-off between complexity and accuracy/modeling dimensionality for a given ACR. It definitely needs to be avoided that complexity is strived for, at the cost of insufficient accuracy or modeling dimensionality. For sure, a result will be obtained, but if it is not trustworthy, it could become completely useless. In this paper, a few basic aspects are mentioned regarding the computational mesh, since this plays a central role in any CFD simulation. Whereas the conceptual discussion in this section also applies to RANS (Reynolds-Averaged Navier-Stokes) calculations, the discussion is devoted to LES (Large-Eddy Simulations), since this turbulence modeling technique is increasingly popular. However, by no means does this suggest that RANS calculations would be inferior. On the contrary, if (classical) LES simulations are performed on ill-suited computational meshes, unsteady RANS calculations can be more reliable, as argued next. In order to explain this, the concept of LES must be briefly recalled, starting from the turbulent energy spectrum [1]. Following the concept of the energy cascade, with large eddies breaking up into smaller eddies and essentially transferring their kinetic energy down to the Kolmogorov scales (i.e., the smallest turbulent eddies, at which level the turbulent kinetic energy is dissipated into heat by viscosity), a turbulent energy spectrum can indeed be constructed (e.g. [1]). Resolving turbulence down to the Kolmogorov scales is not affordable in practice, even in non-reacting flows [1]. Therefore, the first conceptual step in LES is filtering the detailed turbulent flow field, modeling the effect of the 'removed' small-scale eddies on the 'resolved' large-scale eddies. The large eddies are not universal in nature: they depend on the geometry and boundary conditions at hand. Therefore, a major potential advantage over RANS, where all turbulence is modeled, is that these non-universal, and therefore hard to model, large eddies are resolved. In other words, large-scale flow unsteadiness, typical for fire, is resolved. The large eddies, covering the 'energy containing range', indeed contain most of the turbulent kinetic energy [1]. However, using the computational mesh as a filter, as is common practice in CFD calculations in the fire community, this implies that the mesh must be sufficiently fine to resolve the entire 'energy containing range', particularly if the 'constant Smagorinsky' approach is used as subgrid closure. Indeed, one criterion for high-quality LES, developed for isotropic homogeneous turbulence in a box, is that $80 \%$ of the turbulent kinetic energy needs to be resolved [1,2]. If the mesh is insufficiently fine, the fundamental assumption that filtering of the flow field occurs in the inertial range, no longer holds, which is problematic for LES with constant Smagorinsky subgrid closure $[1,2]$. This can be overcome by more advanced subgrid closure techniques, ranging from VLES (Very Large Eddy Simulations) over LES, converging to DNS, depending on the local mesh size (in relation to the local turbulence). Another option is using unsteady RANS: whereas RANS has the disadvantage of stronger dependency of the results on the turbulence model compared to LES, where the large scale turbulent motions ('large eddies') are resolved, RANS has the advantage that coarser meshes can be used, since all turbulence is modeled and the mesh requirement is less severe than for LES. Also, in RANS calculations mesh independence of the results can be obtained by repetitive mesh refinement. For LES by definition this cannot be pursued if the mesh is used as filter [1,2]. However, mesh sensitivity is still a central issue, as discussed next.

As is the case for modeling dimensionality, the requirements on the computational mesh are directly related to the problem one wants to tackle. The criterion most familiar to the fire community, stems from [3], stating that (at least) 10 cells should be taken over $D^{*}$, a characteristic length scale for the fire. This criterion has been established in the context of entrainment processes in smoke plumes above a fire, i.e. to large scale turbulent mixing. In [4], in the context of fire in ventilated tunnels, a discussion on the computational mesh is based on the $80 \%$ criterion for high-quality LES, mentioned above $[1,2]$. Turbulent integral length scales, as obtained from RANS calculations with a model for buoyancy induced entrainment in the RANS model [5], are used in [4] to estimate the required grid cell and, interestingly, the order of magnitude $D^{*} / 10$ corresponds to this in the region dominated by the buoyant plume. 
However, the mesh criterion is not sufficient when, e.g., impingement occurs and boundary layer flows develop. As an example for smoke dynamics, one can think of simulations of activation times of ceilingmounted smoke detectors. To the best of the author's knowledge, no systematic mesh sensitivity study has been published for such configurations. On the other hand, as long as the driving force for the flow is the fire driven buoyancy, the impact on the flow field is expected to be much smaller than on the heat transfer (particularly in the impingement zone, strong mesh dependency of heat transfer can be expected), so the $\mathrm{D}^{*} / 10$ seems reasonable. Yet, situations where buoyancy is overridden by, or at least competing with, forced flows (e.g., mechanical ventilation or vent flows) are very common. In the author's opinion, developing computational mesh requirement criteria for such situations, in combination with wall functions applied, is an important task for the fire safety science community, as further discussed below.

The $D^{*} / 10$ criterion is also not expected to be sufficient when fire dynamics is concerned, with interaction between gas phase and condensed phase phenomena. Flame spread simulations, involving boundary layer flows with convective (and radiative) heat transfer, are strongly mesh dependent [6]. Also simulations of liquid pool fires are currently highly mesh sensitive, particularly when the evaporation model uses the state in the first cell, adjacent to the pool surface, for the mass transfer (burning rate). These issues are addressed in more detail in the section on fire safety science.

\section{THE USE OF SIMULATIONS FOR FIRE SAFETY SYSTEMS DESIGN}

\section{Example from the combustion community}

At CERFACS (Toulouse, France), massively parallel CFD simulations are performed of turbulent combustion in engine applications. Only one recent example is mentioned here, in order to provide some insight on what is possible, but also on what is necessary to obtain reliable results. In [7], Wolf et al. report on a study of azimuthal instability modes, found in annular combustion chambers, with the LES method (also with acoustic solvers, but this is not relevant for the present discussion). The combustion instabilities are mentioned to be difficult to study experimentally, so the correctness of the simulation results is essential, given that engine design will rely upon the insights obtained. Note that, in this sense, the present example could also serve for the next section of the paper, explaining how simulations can be complementary to experiments. In [7], results are discussed of LES of the full aeronautical combustion chamber, representative of a real helicopter engine. The modeling involves the classical Smagorinsky LES approach for turbulence and finite rate (single step) chemistry, using JP10 as surrogate fuel for kerosene. The experimental findings are very well reproduced by the simulation results, i.e. the azimuthal instability models are captured in both frequency and amplitude. The final conclusions of [7] are: '...LES and threedimensional acoustic analysis are powerful tools to investigate combustion instabilities even in complex geometries such as annular chambers. Embedding these steps at an early stage of conception will help creating intrinsically stable combustors.' The question arises why the calculations are successful. First of all, LES, even with the classical Smagorinsky subgrid model, is a good approach to model the turbulence in this design, because the large scale flow instabilities are resolved (and not modeled). The use of the fine computational mesh reduces the importance of the model for the turbulent motions that have been filtered away. Second, single step finite rate chemistry is sufficient to capture the first order chemistry dynamics in the problem at hand. Third, part of the interaction of turbulence and chemistry is automatically resolved, given the fine computational mesh on which the LES calculations are performed. To be brief, a strong investment has been made in the ACR and the RCR has been kept as low as possible, keeping the accuracy and modeling dimensionality high enough (but not unnecessarily high), dealing with the complexity at hand. In other words, the RCR has been set by determining what is necessary to tackle the problem at hand and then ACR has been set equal to, or higher than, RCR. So then the next question is: can this be done in a fire safety system design CFD calculation?

Accepting that a user-defined fire (UDF) is applied (see the next subsection), including a certain soot yield and radiative loss fraction, there is no a priori reason why CFD calculations for smoke dynamics would be more difficult than the example from [7] (as long as combustion products are not considered). On the contrary, unless the geometry is very complex, the simulations for smoke dynamics should be easier, as no compressibility effects need to be accounted for. So RCR is not higher than in the example of [7]. Thus, it can be stated with confidence that CFD, with state-of-the-art models, has the potential of providing very accurate results for smoke dynamics (given a UDF), and therefore can be of great help in the development or assessment of a fire safety system design. However, an important aspect of [7] has not been discussed 
yet, namely the investment in terms of computational mesh. Indeed, in Table 2 of their paper, a size of about 42 million cells is mentioned as a 'coarse' mesh for the full angular calculations, the mesh with about 93 million cells is labeled 'medium' and the label 'fine' is given to a 336 million cells mesh. The mesh sensitivity study of [7] reveals that about 100 million cells are required for accuracy reasons, related to LES, thereby determining the RCR. Obviously, this depends on the problem at hand and the models applied. Yet, to the best of the author's knowledge, these numbers are one or two orders of magnitude higher than what is used at present in the fire community, even in academic research. Sometimes there is a technical reason (i.e., a lower number of cells is acceptable for the problem at hand or thanks to the models applied), but sometimes the reason is time and/or money pressure: results need to be obtained in too short periods of time. In other words: the ACR can be too low to meet the RCR.

Indeed, the practical situation for the fire safety engineer is typically as follows. The problem at hand determines to a certain extent the complexity. One key aspect is the geometry of the building. Indeed, in fire simulations it is never possible to ignore the interaction of flows with boundaries, so the boundaries themselves pose an external imposition on complexity. Also the request for multiple outputs can impose additional complexity. On the other hand, the user can add complexity, depending on the choices made for the type of calculations (CFD or other) or the models used. In any case, the accuracy and modeling dimensionality must be chosen in accordance with the choices made, and with the scenario and objectives for the evaluation of the fire safety system. This altogether determines the RCR. The ACR relates to the available hardware and software, but also to the time frame 'given' by the parties asking for the simulation results, partly determined by economics and/or time constraints. Due to the fact that the lines in Fig. 1 are not quantified to date, it is impossible to give a firm confirmation that ACR $>$ RCR (although, wherever possible, reference should be made to validation and verification work, indicating that ACR $>$ RCR for the simulations at hand). It is thus important, in the author's opinion, to assess the consequences if indeed ACR $<\mathrm{RCR}$. The perception is that simulation results are 'reasonable', but it should be the ambition to solidify and quantify such statements, based on scientific research. If results are not very accurate, it must be evaluated whether at least trends are captured well and what is a reasonable safety margin to apply. If not, simulations can effectively become a burden. This is addressed in more detail in the next section, in the context of bench-marking studies.

The fact that ACR $<$ RCR is possible, also relates to the 'uniqueness' of fire safety from an engineering perspective, in that the proof of the pudding is often not in the eating. Indeed, many fire safety systems never have to operate during their lifetime, so the process of 'learning by doing' (read: learning from mistakes, or, stated more gently, from 'experience') is, to the very best, much slower than in most other engineering disciplines. This is different for the application of the results presented in [7]: once the engine has been designed on the basis of those simulation results, it is preferable for the pilot and passengers that the helicopter engine runs in a stable manner. Nevertheless, it is conceivable to have a prototype engine to validate the simulations with experimental data. Thus, the reliability of the simulation can be tested with the prototype before the simulations are used for design. In fire, the development of a prototype is not an acceptable path thus a reduced system needs to be used for testing the reliability of the simulation. In other words, the accuracy of the CFD simulations (or the reliability of the fire safety system itself) might never be truly tested in real conditions. Establishing the reliability of a fire simulation in its design conditions will therefore require a careful process of extrapolation.

Furthermore, while the simulation results are believed to be a good approximation of reality, because the currently available simulation tools have the intrinsic potential of completeness or accuracy, as illustrated by the vast amount of validation and verification (also beyond the fire community), external constraints (e.g., economics or time restrictions) might reduce ACR, possibly below the RCR (which can be high due to the magnitude of the task). It is therefore of utmost importance to evaluate systematically how reliable simulation results are when using the tool in the way that is perceived as 'common practice' in the fire safety community today. Indeed, accepting that many fire safety systems never have to operate because fortunately often no real hazard occurs during the application's lifetime, our 'engineering experience' for complex cases will rely on simulation results, so they had better be reliable. Note that one could argue that it is more fruitful to force 'common practice' into a systematic quality assessment (QA) system, with systematic verification and validation of the tools (including computer simulations). This is discussed in more detail below in the section on bench-marking studies. Whereas the author subscribes to this point of 
view, reality to date is that such a system is not entirely in place yet, and therefore it is important to try and assess the reliability of current 'common practice'.

[Note that the example refers to CFD with LES, which is common practice for many recent simulation studies in the fire community. This must not be interpreted as a statement that zone modeling or CFD with Reynolds-Averaged Navier-Stokes (RANS) turbulence models would be less valuable.]

\section{User-defined fire}

In numerical simulations in the context of the design of fire safety systems, an important hypothesis concerns the fire source and its location. It is common practice to start from a 'user-defined fire' (UDF), originating from a design fire (e.g. [8]). The size of the fire is imposed in terms of geometrical dimensions and heat release rate (HRR). This can be a 2D source (a burning rate for a fuel, distributed over an area, in combination with a combustion model in the gas phase; or a heat release rate per unit area, over a certain area) or a 3D source (HRR per unit volume over a volume). For obvious reasons, the choice of a 2D or 3D UDF will affect the smoke dynamics close to the fire source and in general, the 'translation' of the assumed fire characteristics into boundary conditions of the numerical model is a very important issue that deserves much attention. However, further away, the differences become smaller, as long as the major driving force for the intrinsic smoke dynamics - the total HRR (and the convective part) - remains the same. If required for the design calculations, chemical species (including soot) can be included. Using the UDF, modeling of the fire dynamics (and, e.g., the effect of water) is effectively avoided. Indeed, the temporal evolution of the UDF is imposed, not calculated. Whereas fire dynamics simulations should remain an important ambition in the fire safety science community (see below), in the context of design calculations there are so many uncertainties in the boundary conditions that the use of a UDF is in fact the only realistic option. Attention can then be focused on other design aspects, such as structural stability or smoke dynamics. Here, it is not intended to provide comments on what values or what temporal evolutions to use for the UDF. The mere observation that a UDF is used in design calculations suffices for the remainder of the present section.

\section{ACR vs. RCR}

If the ACR is increased (ACR $2>$ ACR 1, Fig. 2), it needs to be decided where the investment is put. If it is devoted to higher accuracy, keeping complexity moderate to low, this might be very helpful for theory and model development. Indeed, if accuracy issues are minimized as potential source of uncertainty, the focus can be put on theory and model development. This is discussed in the section on fire safety science below. In the context of design calculations, an investment in accuracy would reduce the level of uncertainty in the simulations. However, the investment is often devoted to increased complexity (e.g., more complex or detailed geometry or more scenarios), given a certain level of accuracy and modeling dimensionality. Note that the graph in Fig. 2 can also be read in another manner: if an external party requires more complexity, given demands on accuracy and accepting a certain level of modeling dimensionality, this implies an increase in the RCR. In other words, a higher level of ACR is required. The quantification of the additional cost involved, based on technical grounds, is another reason to pursue the quantitative evaluation of the curves in Figs. 1 and 2.

\section{State-of-the-art and beyond}

The state-of-the-art for fire safety systems design calculations is CFD. Whereas time pressure is omnipresent, the calculations do not need to be faster than the time scales in evolving fires in reality (in contrast to forecasting calculations, described below), so CFD is affordable and therefore typically preferred over two-zone modeling. However, this does not imply that zone modeling is to be ruled out as a valuable tool. Indeed, being very fast, zone modeling is useful for a preliminary order of magnitude analysis, which is helpful for the determination of e.g. extraction flow rates in a CFD simulation afterwards. Also, the combined use of CFD where necessary (e.g. where detailed info is required or where the flow field is complex) and zone modeling where possible (e.g. in essentially one-dimensional flows), is a very smart way to optimize the ACR and reduce the RCR. One example of such an approach, combining CFD with network calculations for tunnel networks, is described in [9].

In CFD simulations, the use of a UDF is the only feasible option to overcome the uncertainties in boundary conditions (not in the least in the fire evolution itself). In the design phase, there is no way to circumvent 
the uncertainty in the circumstances of the possible real fire, so there is no need to try and simulate complex fire dynamics in terms of coupled CFD - pyrolysis calculations (except perhaps in very special circumstances). Obviously, validation, verification and a sensitivity analysis of the simulation results on the choices made, are all extremely important actions (see, e.g., [10]).

The state-of-the-art is that, conditional upon a sufficient level of accuracy and a correctly defined UDF given boundary conditions (which may involve a smoke control system), CFD calculations are reliable with respect to the question of whether or not smoke is present at a certain location at a certain moment in time. In other words, the binary 'yes/no' question can be answered. Note that smoke can even be treated as hot air (with a tracer) in the simulations. The smoke concentration (e.g., in $\mathrm{g} / \mathrm{m}^{3}$ ) can also be calculated in a reliable manner, provided that the source term is prescribed, e.g., by making the smoke production rate proportional to the burning rate. Once this is accepted, the problem is reduced to a convection - diffusion equation. To what extent the basic assumption resembles reality, though, is another issue. Indeed, in the author's opinion, soot modeling should remain high on the priority list of tasks for the fire safety science community, as discussed below.

With respect to species, an effort should be made towards inclusion of $\mathrm{CO}$ in the calculations. To the best of the author's knowledge, much testing and validation work still needs to be performed. Particular issues are the possibility of under-ventilation, but also the relatively low temperatures, observed in fires. These temperatures are often outside the validity domain of chemistry mechanisms, developed in the combustion community, where typically higher temperatures are envisaged. However, ongoing research in lowtemperature combustion for reduced emissions (e.g., $\mathrm{NO}_{\mathrm{x}}$ ) may provide valuable input.

Another interesting aspect concerns the effect of water, be it in the form of sprinklers or water mist systems. At the level of interaction with the fire source itself, a reduction in size (geometry and HRR) of the UDF is a reasonable approach, given all other uncertainties. At the level of interaction with smoke, a downward smoke layer displacement can be considered (see e.g. [11]). Yet, to the best of the author's knowledge, this is not yet common practice in the fire safety science and engineering community.

\section{THE USE OF SIMULATIONS FOR PROGRESS IN FIRE SAFETY SCIENCE}

\section{Theory and model development}

Numerical simulations can be a very helpful tool in the development of theory and models in fire safety science. Or, using Torero's terminology: ‘... the use of CFD as a basis for the Scaling-Up of fire has a very clear gain and therefore there is a strong motivation for the development and improvement of these tools' [12]. The question remains: what route needs to be followed?

Starting from Fig. 2, the decision can be made to invest the ACR as much as possible in accuracy. Clearly, this is only possible for 'simple' configurations. However, investing in accuracy may allow ruling out the need for modeling and therefore the related uncertainties. At the level of the turbulent flow field, translating the increase in accuracy as computational mesh refinement, the evolution is made towards 'Direct Numerical Simulations' (DNS). It is well-known (see e.g. [1]) that the mesh size must be smaller than the smallest turbulent length scale (i.e., on the order of the Kolmogorov length scale). In a way, such consecutive mesh refinement, more and more possible thanks to evolutions at the computer hardware level, can be compared to increased resolution of cameras in experiments. Just as increased camera resolution allows analysis of more and more detail, DNS allows investigating more detailed phenomena. In fact, more detail can be reached numerically than experimentally and specific aspects can be examined. DNS is limited to low-Reynolds number flows, due to strongly increasing computational costs with increasing Reynolds number [1], but computer hardware developments, as well as algorithmic developments in e.g., adaptive mesh refinement (AMR), continuously push the limits upward. Whereas the amount of data is huge and one has to focus on what one wants to examine, once the database is established, it contains a wealth of information that can be useful for further model development. This is also true for DNS with combustion (where additional issues can be raised, concerning the completeness of the combustion modeling, but that discussion is beyond the scope of the present paper). A recent example of advances in combustion modeling, using DNS databases, is worth mentioning, as it discusses finite-rate chemistry aspects, a topic that might be relevant in the context of resolving species (see below) [13]. In this sense, CFD (in the form of DNS) can be labeled 'indispensible' for future theory and model development. 
Yet, the question is whether the fire safety science community should consider doing DNS. Clearly, it is not possible to perform DNS for real-life fire calculations and therefore models are in place. This can be done, starting from DNS results and/or data bases. For the development of turbulence and combustion models, in the author's opinion, it is more effective to 'import' progress in those fields into the fire community, than to try and go through the same development process. Indeed, many fire-induced flows are low to moderate Reynolds number buoyancy driven flows with non-premixed combustion. Therefore, models for turbulence, combustion, TCI, radiation, TRI are fundamentally no different from what is developed in the turbulence and combustion community. However, DNS work could still be very valuable for low-Reynolds number boundary layer flows with heat and mass transfer, ubiquitous in fire. Other typical fire aspects, making DNS useful (also for further theory development), are the Froude number range (in between combustion and numerical weather modeling) and the absence of a clear inertial range in turbulence. In addition to such DNS, in the author's opinion, an investment in extensive fire dedicated bench-marking studies, bringing in the uniqueness of fire, is more effective and in fact indispensible for the further development of theories and models in fire safety science. This is discussed further in the next subsection.

Before doing so, a brief discussion is devoted to TCI and TRI, because they are examples of what can be imported from the combustion community. Indeed, these concepts are very well known and have been studied extensively in the combustion community, but to the best of the author's knowledge, they are not often mentioned explicitly in fire simulations. TCI has been a central issue in the TNF workshop series (see below) [14]. As turbulence interacts with combustion, a 'Probability Density Function' (PDF) needs to be used. Particularly when finite-rate chemistry effects are important, such as for ignition or (local) extinction problems, 'scatter' is observed in experimental measurement data for chemical species and temperature as function of mixture fraction in turbulent reacting flows (see e.g. $[15,16]$ ). TCI can be incorporated through a presumed PDF, but also through the transported PDF technique [17] (among other possibilities, such as the eddy dissipation concept (EDC) to handle turbulent mixing with fast chemistry, or the 'discrete quadrature method of moments' (DQMOM) to handle subgrid heterogeneity). The computational cost of the latter is much higher, but has been reduced substantially through optimization techniques and has become more and more tractable thanks to computer hardware developments. The effects of TCI are more influential in RANS calculations (as all turbulence is modeled there), but it can be worth-while investigating the potential of the transported PDF technique in LES as well. This is certainly the case when finite-rate chemistry is relevant, which can be expected for the relatively low temperatures encountered in fires and more precisely for toxicity calculations (such as CO). An example of state-of-the-art LES/PDF calculations, involving soot, is found in [18], while a recent review on the progress in PDF methods is found in [19].

TRI received less attention in the TNF series because the study focused on laboratory-scale flames. Yet, this could be a very important issue in the context of fire. Indeed, the interaction of turbulence with radiation can strongly affect the value of the maximum temperature on the one hand, but also of the radiative flux on the other hand. This can be important concerning thermal attacks on people and structures. Without going into further detail, TRI concerns the fact that the average value of the fourth power of temperature is not the same as the fourth power of the average temperature, and that fluctuations in (gas) absorptivity are correlated to temperature fluctuations, so the product of the averages is not the same as the average of the product. Again, TRI is much more important in RANS than in LES. Still, even in LES this can be an issue. A recent overview paper on this topic is found in [20].

\section{Bench-marking: A collaborative joint effort among experimental and numerical researchers}

As mentioned above, bench-marking studies are considered indispensible in the process of making progress in fire safety science (and, by inference, fire safety systems designs), taking advantage of computer simulations. It must be acknowledged that many extremely valuable efforts are already ongoing. One important workflow concerns the systematic 'Verification and Validation' (V\&V) effort in the context of the development of FDS. This process, along with the repository, is open to anyone who wants to contribute (although, to date, the response from the community is 'disappointing', according to https:/groups.google.com/forum/\#!topic/fds-smv/jmIyOy2wzFA). The key target of this V\&V effort is making the FDS CFD code more 'fail-safe', improving numerical aspects and modeling reliability. Obviously, this is very beneficial for the fire safety science community. A different approach is taken in the 
'FM Global Open Source CFD Fire Modeling Workshop' series, where the key target is 'to develop the capability of predicting fire growth behavior and fire protection requirements within the framework of physics-based models', according to https://sites.google.com/site/firemodelingworkshop/ This workshop series concerns a combination of experimental and numerical efforts, with the intention of improving fire modeling in simulations, using the OpenFOAM open source CFD platform. The multi-physics aspect is given much attention, which is logical and relevant in the context of fire safety science.

Both routes are very valuable and worth pursuing and intensifying. Yet, in the author's opinion, it is worthwile to identify and/or define a number of 'target' test cases, putting development of fire safety science as 'key target', with improvement of fire modeling and simulation codes as very valuable 'side product'. This is in no way conflicting with the ongoing efforts. Rather, it is of added value and should lead to very valuable cross-fertilization of all V\&V efforts mentioned. Two examples from the combustion community are briefly discussed in this light, because in the author's opinion, they are enlightening examples of the way to set up bench-mark studies on the one hand, and of the creation of a collaborative community of experimentalists and modelers on the other hand. This has clearly led to efficient, rapid and systematic progress in knowledge development.

The first example concerns the series 'International Workshop on Measurement and Computation of Turbulent Nonpremixed Flames' (http:/www.sandia.gov/TNF/abstract.html), known as the 'TNF Workshop' series [14]. The first sentence on the website reads: 'This workshop is an open and ongoing international collaboration among experimental and computational researchers in turbulent combustion.' The collaboration among experimental and computational researchers, along with openness in discussions, is fundamental. It is not at all a competition. The ever ongoing intense and open communication pushes the experimentalists to set up and characterize the experiments such that the level of uncertainty at the level of material (fuel) properties and boundary conditions is minimized as much as possible. They are also pushed to measure quantities that are deemed relevant by the modelers. At the same time, the modelers are pushed to improve the models through continuous evaluation of the models' accuracy, keeping in mind the required numerical accuracy. They are also forced to make sure that all calculations are documented such that they are reproducible, an essential feature in a validation process. Interestingly, insight in turbulence chemistry interaction phenomena evolved much more rapidly than what would be possible from examining experiments or simulations alone. Indeed, taking advantage of ever increasing computing power, some phenomena were observed first in the simulations and confirmed afterwards in experiments (and vice versa, of course).

A second important aspect is that the data for a wide range of flames is publicly available. This range grew over the years, starting from jet flames with light hydrocarbon gas fuels in the non-premixed regime. Having gained confidence in the models, which improved over the years, steps have been taken towards more complex configurations (including e.g. swirl), other fuels and other combustion regimes. The essential point is that the step-wise approach has led to cases that are very well characterized and at the same time of practical relevance for real-life problems.

Another important aspect is that a range of flames is considered, as opposed to a single test case. This is crucial, because tuning of models to obtain as close agreement as possible for a single problem is far less valuable than the development of models that are reliable in predicting trends correctly. Studying a range of flames also assists in defining ranges of applicability of models.

A similar effort has been initiated more recently in the context of turbulent spray combustion [21] (http://www.tcs-workshop.org/). The philosophy is the same as in the TNF workshop series, but the fuels are liquid, so additional problems like droplet break-up and evaporation pop up. Again, ranges of flames are studied, not one single flame.

There is no technical reason why a similar undertaking, with target test cases, would be impossible for the fire safety science community. The systematic evaluation of the accuracy and reliability of CFD simulation results with state-of-the-art models as used now and with typical numerical settings (including typical computational mesh cell size), should indeed remain high on the priority list and could be crystallized into target test cases. A range of configurations and, within each configuration, a range of conditions should be investigated in order to assess whether trends are captured correctly. This is more important than a perfect match between simulation results and measurement data for one specific case. The configurations should be such that they allow complete characterization of boundary conditions and accurate measurements. They 
should target an aspect of value to fire simulations and should be set up such that only this aspect is assessed, without intermingling from other parameters. This is in line with [12]. Whereas some suggestions are made below, the configurations need not all be newly invented. Existing set-ups can be revisited with modern experimental techniques and up-to-date computing power. This is a tedious task and a joint effort of experimental and simulation researchers. This cannot be left to be done by individual research units. Therefore, and also to guarantee user independence, in the author's opinion, this effort should be coordinated by the fire safety science community. Such coordination and the crystallization of the efforts towards 'target cases' could make the response from the community to ongoing V\&V efforts less 'disappointing' (see above).

The outcome of such continuing effort would be extremely valuable, in that it would provide quantitative information for the graphs in Figs. 1 and 2. Interestingly, this also relates to important points, raised in [22]. Indeed, in that review paper on fire modeling, one aspect claimed to be 'hindering ... to move forward is the increasing level of miscommunication between modelers and experimentalists, scientists and engineers, ...' [22]. The bench-marking study in the format and spirit as described would effectively make sure that modelers and experimentalists interact more closely, thereby effectively reducing possible miscommunication. Moreover, if chosen carefully, the bench-mark test cases have the potential of 'bridging the gap' between scientists and engineers, another point raised in [22]. In doing so, this should also add value to the ongoing $\mathrm{V} \& \mathrm{~V}$ efforts mentioned above, stimulating the progress already made since [22].

To conclude this section, it is also recalled that reproducibility is an essential feature in any validation related effort. This holds for the experiments as well as for the simulations.

\section{Examples of possible bench-marking studies}

As mentioned, the bench-marking studies should, in the author's opinion, keep 'fire' as central application topic. In other words, focus should be on an aspect that is relevant to the fire safety science community and the study must be set up to investigate that aspect in isolation. Knowledge and experience obtained during developments in other communities should be incorporated in a systematic manner, where possible. In this subsection, a humble suggestion is made for a few possible bench-marking studies. Only the first suggestion is elaborated to some level of detail.

The first suggestion concerns the systematic study of the loss in accuracy when using (too) coarse meshes in CFD simulations. This is typical for fire simulations, due to the boundary conditions: the large and complex geometries in, e.g., a multi-compartment fire force us to use meshes with cells that are at least an order of magnitude larger than what can be afforded in, e.g., detailed turbulent flame calculations. Consequently, the reaction zone is not captured at all and turbulence modeling becomes less accurate (particularly in an LES context). Also, TCI is not automatically captured. Therefore, in the author's opinion, a systematic assessment how strongly the accuracy and reliability of results decreases when the computational mesh becomes coarser, keeping all other simulation settings identical, is an important task. In academic studies, a mesh sensitivity study is often presented, but typically this is limited to justifying why in the end a certain cell size has been chosen. Systematic quantification of deviations is sometimes added, but even then, the study is obviously limited to the problem at hand. Pursuing such a mesh sensitivity analysis in the context of a bench-marking study, leading to the identification of acceptable mesh sizes under certain circumstances and to the quantification of deviations between results obtained with different mesh sizes, is a very tedious and cumbersome task, but the return on investment is a tremendous step forward in the maturing process of the use of simulation tools in fire safety system design.

One possible example for such a study concerns flame-wall interaction, relevant for flame spread simulations (Fig. 3). Boundary layer flows occur with convective (and radiative) heat transfer. Such simulations are strongly mesh dependent, as reported in, e.g., [6], where wall-resolved LES simulations are discussed. Wall-resolved LES simulations require very fine computational meshes, but, for the sake of research purposes, are worthwhile doing for a range of test cases, generating 'reference results'. Knowing that there is strong coupling between heat transfer from the (typically) turbulent sooty flame and pyrolysis, and appreciating that there is still uncertainty in the modeling and measuring of all phenomena involved, it can, in the author's opinion, be argued to use numerical simulation results as 'data set', rather than to try and use experimental data in this respect. There is simply too much uncertainty to allow pinpointing where 
exactly modeling or accuracy needs to be improved to date. This is in line with the statement that '... wallresolved simulations are considered a necessary first step on the route to develop wall models', in the context of turbulent vertical wall fires [6]. Nevertheless, one could think of an experiment as well, using a gas burner (with, e.g., a long narrow slit nozzle) from which gaseous fuel emerges at low velocity (so the set-up is buoyancy driven). Different fuels (e.g., methane, propane) and a range of fuel velocities should be applied. Making the fuel gaseous allows for control of the HRR. Choosing non-sooty fuels reduces uncertainty at the level of radiation and soot formation. It is essential to measure fields of temperatures, species and velocities (including turbulence) in the gas phase in a non-intrusive manner, both for the configuration of a 'free' flame and the situation where the flame is placed attached to the plate. Temperatures and heat fluxes at the plate surface must also be measured. The study could be limited to inert plates first, in order to rule out pyrolysis phenomena.

The following step-wise approach could be evaluated in the simulations, with the objective in mind of assessing the accuracy of simulation results obtained on coarse meshes:

- Highly resolved LES free flame simulations serve as starting point. A flamelet type combustion model (even with a single strain rate or scalar dissipation rate) could be used as first step. Different fuel supply rates can cover a range of conditions (yet, the configuration should remain buoyancy driven, i.e. relevant for fire).

- Subsequently, this flame can be put at the bottom of an inert vertical plate. The flame shape will of course vary, but more importantly the flow field and heat transfer to the plate must be studied.

- Next, the mesh should be made coarser in the gas phase for the configurations mentioned and changes in maximum temperature value and position must be quantified. It must also be identified whether or not the mesh can become unacceptably coarse (and if yes, the conditions must be quantified).

Although the assumption of infinitely fast combustion might no longer be valid, the flame can also be put underneath a horizontal plate, so that an impingement configuration is created. In that case, it is probably better to start with an axisymmetric flame, rather than a two-dimensional flame. The same steps can be taken as just described, for different distances between the plate and the fuel nozzle.

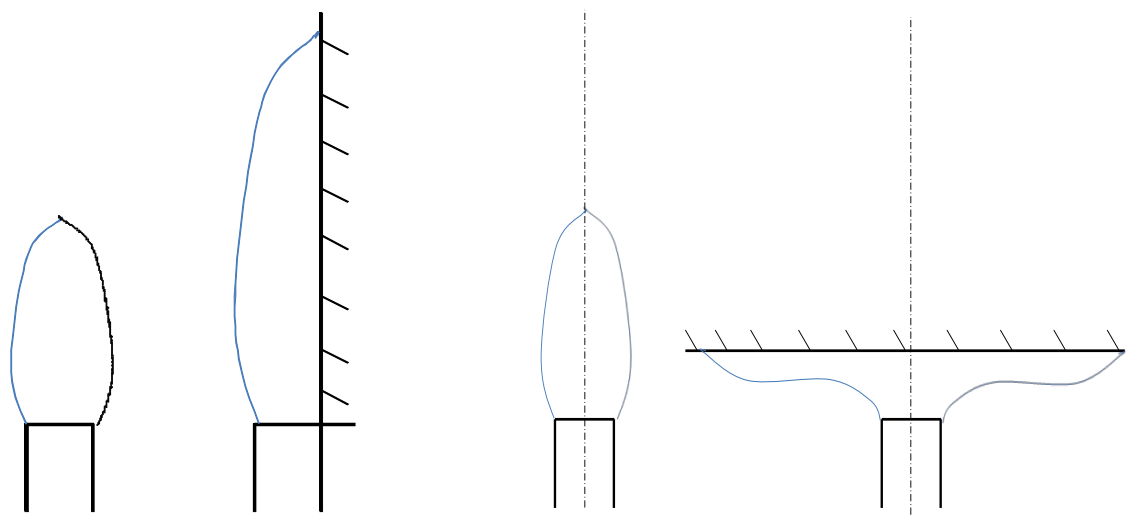

Fig. 3. Schematic of a step-wise approach to investigate computational mesh requirements. Left: 2D configuration, relevant for upward flame spread. Right: axisymmetric configuration, relevant for impingement situations and flows underneath ceilings.

Ideally, this effort is taken up by multiple independent users of different software packages (with multiple users per software package). Note that this effort concerns both LES and RANS type calculations. Note also that there is existing literature in the turbulence and turbulent combustion community. However, the flows examined are typically momentum driven, rather than buoyancy driven, so there is value in doing a study as described above. Note also that soot modeling, radiation and pyrolysis modeling have not been mentioned. Obviously they are all important (see next subsection), but presumably their modeling issues are less directly related to requirements on the CFD computational mesh (provided it is fine enough to capture the maximum temperature accurately).

A second suggestion for bench-mark studies concerns multi-compartment fires at reduced scale (so that the experiments are affordable). The multi-compartment aspect, with strong interaction between flames (combustion) and solid boundaries (walls, ceilings) is again typical for fire. A gas burner (e.g. with propane 
as fuel) should be used, so full control of HRR is guaranteed. One could think of a fire in a room, adjacent to an atrium, with only a door opening in between. Depending on the HRR and the door opening size, the fire is well-ventilated or not. In the latter case, flames can emerge into the atrium. If another floor is put on top of the original room, vertical fire spread can be studied. Also leakage at the ceiling level can be examined. Again, it is important to measure fields (including higher order statistics) of temperatures, species and velocities (including turbulence) in the gas phase in a non-intrusive manner, as well as heat fluxes. From a simulation point of view, the step-wise approach mentioned above could again be adopted. Making the experimental set-up modular would open up a range of possibilities.

A third suggestion concerns a gaseous burner, placed in a corridor or a tunnel that is closed at one end. The burner should be placed close to the closed end (but far enough to avoid flame-wall interaction). Further, a sprinkler head (or a water mist nozzle) should be placed in between the open side of the corridor/tunnel and the burner. Fields of temperatures, species and velocities (including turbulence) should be measured in the gas phase in a non-intrusive manner, as well as heat fluxes. After some time, the water system should be activated and the effect is measured on the flow field (e.g. downward smoke layer displacement) and on the fire dynamics. From a simulation point of view, the step-wise approach mentioned above could be adopted.

\section{State-of-the-art and beyond}

As mentioned, CFD calculations of smoke dynamics where the fire and the soot yield are prescribed, are state-of-the-art (see e.g. [23]). However, as soon as more detail is required (e.g. species or visibility issues), combustion and heat transfer play an important role. This is a fortiori true for fire dynamics simulations. A few suggestions are made here for priorities for the near (and longer term) future in the context of computer simulations in the fire safety science community. The basic point of view is that the application 'fire' should remain central in research activities in the fire safety science community. For several phenomena, including turbulence, combustion and radiation (and other types of heat transfer), developments made in other research communities should be 'imported'. However, care must be taken when importing knowledge from other fields into the fire community, because 'scaling up fire' is far from straightforward [12].

There are indeed a number of aspects that are quite particular for fire, so it cannot be expected from other communities to dedicate research activities to those phenomena (which is why the fire safety science community should pick those up). First of all, soot modeling should remain high on the priority list of tasks for the fire safety science community. It is well-known that soot is directly related to optical thickness/visibility. It strongly affects the maximum temperatures observed, because it is dominant with respect to radiation, and it affects the formation of CO. Soot is also of interest for the combustion community and much research is ongoing. Yet, it remains to be seen to what extent observations and models from the combustion community remain valid in the case of fire, particularly when combustion takes place in low temperature regimes without sufficient ventilation, or in vitiated conditions (e.g. combustion inside a smoke layer). Vice versa, models based on the 'smoke point', such as [24], are useful for fire simulations, since they rely upon experimental data from circumstances that are relevant for fire configurations. Yet, they are not directly useful to predict soot levels in momentum-driven flames.

Second, the effect of water (from sprinklers or water mist systems) must be studied systematically. This must be done at the level of interaction between water droplets and the fire source or the flames, but also at the level of interaction with a smoke layer. Indeed a downward displacement of a still or horizontally moving smoke layer will occur under the effect of water. An analytical model has been suggested [11], but many more CFD simulations, including sprinkler and water mist nozzle distributions, should be performed to increase insight. The smoke can also move vertically, in a buoyant smoke plume, and the effect of water should be studied in CFD simulations for this scenario as well. For studies of interaction with smoke, hot air can be used in order to rule out as many uncertainties as possible. It is essential to develop experiments where ranges of parameters are examined in a complete and accurate manner, with carefully defined and characterized boundary conditions.

An effort should also be made towards inclusion of $\mathrm{CO}$ in the calculations. Two important aspects are involved. First of all, CO is known as the major cause of fatalities in fire. Second, CO is combustible and therefore, being present in smoke layers, it is a severe threat for fire fighters during intervention. Indeed, smoke layer ignition is life threatening. It is crucial to build knowledge and experience on both of these aspects. To the best of the author's knowledge, much testing and validation work still needs to be performed. Particular issues are the possibility of under-ventilation, but also the relatively low 
temperatures, observed in fires, and the vitiated conditions in the smoke layer. This can be outside the validity domain of chemistry mechanisms, developed in the combustion community, where typically higher temperatures are found. However, the ongoing research in low-temperature combustion in an attempt to reduce $\mathrm{NO}_{\mathrm{x}}$ formation may lead to valuable input for the fire community.

As the paper focuses on gas phase phenomena, little has been said on pyrolysis. Still, pyrolysis modeling deserves continued attention from the fire safety science community. Pyrolysis in the context of fire is not the same as in combustion devices. The author believes that the idea of pyrolysis fronts (see e.g. [25]) should be further explored in the context of flame spread simulations, whereas at the same time very 'complete' pyrolysis model packages like [26] must be further exploited, not only to generate reference solutions, but also for flame spread simulations (in combination with parameter optimization techniques).

Finally, it would be interesting for the fire safety science community to undertake an effort in the domain of liquid pool fires, where currents inside the liquid pool are simulated. Their existence and the fact that convective heat transfer from the fuel surface is non-negligible are well known. Therefore, to the author's opinion, it would be a valuable exercise to perform CFD calculations and systematically analyze the impact of neglecting such currents. This would be a strong step forward in the context of liquid pool fires.

\section{Different types of experiments}

As explained, the author thinks there is a strong need for bench-marking studies on sufficiently simple configurations that yet are sufficiently complex for practical purposes. However, there will also remain a strong need for repetitive small-scale experiments and for full-scale experiments. This can be put into perspective from the philosophical 'walk before you run' principle.

Repetitive small-scale experiments, on which statistical analyses can be performed, remain extremely useful and valuable for model development and validation. Along with the bench-marking ('intermediate' scale) configurations, this can be interpreted as an effort to master and further develop the walking process, to identify what is solid ground to walk and run on, and to evaluate what is the risk of falling when running (read: how reliable does the result remain for a real-life full-scale configuration). In other words, these types of studies are important in order to build up knowledge and experience in walking processes, as basis for the running process and to improve the running skills. Yet, concerns can be raised, given the complexity of fires, to what extent small-scale tests relate to real fires and how the information collected can be translated into model parameters in simulations that are intended to tackle real-life problems. Therefore, in the author's opinion, academic researchers also need to run from time to time and learn from possible crashes in doing so (read: learn from discrepancies between simulation results and measurements), so the engineer can learn from those crashes. Expensive as that might be, it is a worthwhile investment on the long term to pursue small-scale, intermediate-scale and full-scale set-ups, because they all provide valuable and complementary information for the engineer, who has no other choice than to run. Examples of recent 'realistic' full-scale experiments are the 'Dalmarnock' tests [27], car park fire tests [28,29], the 'Rabot' apartment fire tests [30] and the PRISME tests [31]. A complex problem is tackled and the experiments and simulations are set up with great care, such that one can learn from 'crashes' while running. This is extremely important in the process of learning, since, as mentioned before, many fire safety systems do not have to operate during their lifetime, so there is only a very slow process of 'learning by doing' in the real world. Therefore, thoroughly analyzed 'crashes' are essential for the long-term progress of fire safety science and engineering. Yet, for the sake of completeness it is recalled that reproducibility can be an issue in full-scale tests, despite characterizing them as carefully as possible. At the same time, simulation results from deterministic models depend on the boundary conditions used. In that sense, the spreading of simulation results and deviations from experimental observations as reported in [32] for the Dalmarnock tests must not be interpreted as 'discouraging'. Rather, the analysis must be pursued to evaluate whether the deviations are caused by shortcomings in the set-up of the numerical simulations or not. As final comment, it is mentioned that 'running before walking' can pose a major problem in that full-scale experiments cannot be disassembled and causality can no longer be established [12]. If that is the case, it is hard to "learn" from them. This again illustrates the importance of bench-marking studies as mentioned. 


\section{THE USE OF SIMULATIONS FOR FIRE FORECASTING}

The domain of fire forecasting will play a central role in the fire safety science community in the coming decades. Therefore, some discussion is devoted to this topic. Fire forecasting calculations are extremely valuable for fire service intervention assistance and for firefighter safety. The idea has been launched at The University of Edinburgh in the 'FireGrid' project (see, e.g., [33]). The present paper focuses on fires in enclosures, but the domain of fire forecasting obviously extends to wildfires and wildland - urban interface (WUI) fires as well (see, e.g., [34]).

\section{The need for speed}

By definition forecasting simulations must be faster than real-time. Thus, there is a clear need for speed. Current levels of ACR and RCR render the use of CFD for forecasting purposes practically impossible for the time being. A two-zone modeling approach is a far more time efficient solution. One could think of performing CFD on a very coarse mesh. However, the key advantage of CFD over two-zone modeling, namely the fact that the momentum equations are solved and consequently flow phenomena can be captured, is lost if there is no accuracy in solving the momentum equations. Therefore, in the author's opinion, it is better to use two-zone model calculations and remedy deviations by taking advantage of sensor data (see the following subsection) to date. Note, in this respect, that the uncertainties in boundary conditions (including the fuel and fire heat release rate) remain, also in CFD calculations, so there is a need for assistance by (sensor) data 'on the fly' during the calculations anyway. The analogy to numerical weather predictions (NWP) is apparent, so many aspects can be learnt from NWP, particularly with respect to data assimilation assisted simulations.

\section{Sensor-assisted simulations}

As mentioned, supply of data, gathered by sensors, is necessary during the simulations, in order to circumvent uncertainties in boundary conditions. Indeed, there would still be a user-defined fire, in combination with thermal (and ventilation) boundary conditions, very much like what is done in fire safety design calculations. However, the key difference is that the UDF is varied on the run, taking advantage of sensor data, monitoring the fire as it evolves (as described in e.g. [35]).

The fire forecasting concept involves an optimization part to find the fire parameters (e.g. size and location) to obtain the best match between the sensor data and calculation results. This is an inverse problem and several Inverse Fire Modeling (IFM) frameworks have already been developed for a number of fire scenarios, using different optimization techniques. The most important parameter to be estimated is the HRR (in combination with thermal (and ventilation) boundary conditions). In most frameworks [35-39], this is performed by analyzing temperature and/or smoke measurements. An alternate approach has been developed in [40,41], reconstructing the HRR from video recordings of flame dimensions obtained with the means of a flame detection algorithm. Such video data could stem from surveillance cameras. Multimodal techniques, incorporating long-wave infra-red images in addition to visual data, seem a promising approach to overcome difficulties when cameras are immersed in smoke [40,41]. A projection of the HRR is then fed into a two-zone model to produce forecasts of upper layer temperature and smoke layer height.

As stated in the previous subsection, predictions with positive lead times are a conditio sine qua non for fire forecasting calculations, so the efficiency of the IFM framework is important. This efficiency depends on the optimization (i.e. inverse modeling) procedure and different methods are described in [35-41]. Further optimization and testing will undoubtedly be performed during the coming years.

\section{CONCLUDING REMARKS}

It has been argued that fire simulations are of unique value in different respects: for fire safety system design calculations, for improving our understanding (theory and model development and validation) and for fire forecasting. In all areas, much work still needs to be done.

A discussion has been devoted to the relation between ACR (Available Computing Resources) and RCR (Required Computing Resources). It is important to establish the accuracy, complexity and modeling dimensionality required and to carefully map it to what can be achieved with available resources. In other words, the investment in complexity, accuracy and modeling dimensionality must be done with great care. 
Making the ACR and the RCR more quantitative and assessing the consequences if ACR $<$ RCR are considered major and imminent tasks for the fire safety science community.

Fully resolved combustion simulations clearly exceed the current ACR and will remain so in the foreseeable future. Also, flame and fire spread simulations and extinction, for example by water, are hard to perform. Therefore, CFD simulations in fire safety design calculations will continue to rely upon a userdefined fire. Thus, it is a worth-wile effort to try and establish a proper user-defined fire.

Improvements in CFD fire simulations can be made by carefully importing knowledge and progress from other research communities. However, certain aspects are fire specific and the fire safety science community is encouraged to take up the task of resolving issues that are only relevant for fire. It is suggested that bench-marking studies at all levels and scales should play a central role in this effort, in order to evaluate in a systematic manner the reliability of CFD simulations of fires, with special attention to cell sizes in the computational meshes used. This should not be done for single isolated problems, but for a range of configurations and, within each configuration, for a range of conditions. This is essential, because it is more important to capture trends correctly than to have a perfect match between simulation results and measurement data for one specific case. The configurations should be chosen such that they allow focusing on a single aspect, relevant for fire, with as full as possible characterization of boundary conditions and as complete and accurate as possible measurement data. These configurations do not need to be all newly invented. There can be high value in revisiting set-ups with modern experimental techniques and up-to-date computing power. As this is a tedious task and supposed to be a joint effort of experimental and simulation researchers, and the outcome should be user independent, the author considers this a task to be coordinated by the fire safety science community. This way, the indispensible communication between modelers and experimentalists, and between scientists and engineers, can also be further stimulated.

The time frame is estimated to be in the order of (at least) a decade, for systematic progress to be made and distributed into the fire safety science and engineering community, once the target test cases for a devoted joint bench-marking study have been agreed upon. Yet, the author firmly believes this is an effort worth the investment, particularly as the exercise is not purely academic in nature. Indeed, in the absence of 'past experience' with fire safety systems, there is a need to build up engineering experience, using simulations. In that respect, quantification of the reliability of the simulations or of the required safety margins is essential.

It is recalled that the paper does not intend to suggest that all research effort in the fire community must be devoted to gas phase phenomena. On the contrary, much effort is required in the multi-phase aspects in fire dynamics (pyrolysis modeling, fire suppression, interaction of water with flames and smoke, etc.), since progress in modeling the multi-phase phenomena will strongly add to progress in fire safety science.

Finally, it is emphasized that, in the author's opinion, sensor assisted fire forecasting simulations will play an important role in the decade to come, and should therefore be pursued as part of the structured effort to improve the value that fire simulations have for society.

\section{ACKNOWLEDGMENTS}

The author sincerely thanks all the colleagues and researchers with whom there has already been some form of collaboration. The author also acknowledges his colleagues and friends who read this paper in its early form and provided valuable feedback, leading to the above version.

\section{REFERENCES}

[1] Pope, S.B., Turbulent Flows, Cambridge University Press, 2000.

[2] Pope, S.B. (2004) Ten questions concerning the large-eddy simulation of turbulent flows, New Journal of Physics (article 35). http://dx.doi.org/ 10.1088/1367-2630/6/1/035

[3] McGrattan, K.B., Baum, H.R., and Rehm, R.G. (1998) Large eddy simulations of smoke movement, Fire Safety Journal 30 (2): 161-178. http://dx.doi.org/10.1016/S0379-7112

[4] Van Maele, K., and Merci, B. (2008) Application of RANS and LES field simulations to predict the critical ventilation velocity in longitudinally ventilated horizontal tunnels, Fire Safety Journal 43: 598-609. http://dx.doi.org/10.1016/j.firesaf.2008.02.002 
[5] Van Maele, K. and Merci, B. (2006) Application of Two Buoyancy-Modified k- $\varepsilon$ Turbulence Models to Different Types of Buoyant Plumes, Fire Safety Journal 41 (2): 122-138. http://dx.doi.org/10.1016/j.firesaf.2005.11.003

[6] Ren, N., Wang, Y., Vilfayeau, S., and Trouvé, A., "Large Eddy Simulation of Propylene Turbulent Vertical Wall Fires", $7^{\text {th }}$ International Seminar on Fire and Explosion Hazards, 2013, pp. 252 - 261. [ISBN: 978-981-07-5936-0]

[7] Wolf, P., Staffelbach, G., Gicquel, L.Y.M., Müller, J.D. and Poinsot, T. (2012) Acoustic and Large Eddy Simulation studies of azimuthal modes in annular combustion chambers, Combustion and Flame 159: 3398-3413. http://dx.doi.org/10.1016/j.combustflame.2012.06.016

[8] van Hees, P., "Challenges for international standardization during the next decades with respect to the areas of new innovative products and fire safety engineering", Fire and Materials Conference, 2013, pp. 803 - 809 (Interscience Communications, London).

[9] Colella, F., Rein, G., Borchiellini, R., and Torero, J.L. (2011) A Novel Multiscale Methodology for Simulating Tunnel Ventilation Flows During Fires, Fire Technology 47 (1): 221-253. http://dx.doi.org/10.1007/s10694-010-0144-2

[10] van Hees, P. (2013) Validation and verification of fire models for fire safety engineering, Procedia Engineering 62: 154 - 168. http://dx.doi.org/10.1016/j.proeng.2013.08.052

[11] Tang, Z., Vierendeels, J., Fang, Z., and Merci, B. (2013) Description and Application of an Analytical Model to Quantify Downward Smoke Displacement Caused by a Water Spray, Fire Safety Journal 55: 50 - 60. http://dx.doi.org/10.1016/j.firesaf.2012.10.012

[12] Torero, J.L. (2013) Scaling-Up fire, Proceedings of the Combustion Institute 34: 99-124. http://dx.doi.org/10.1016/j.proci.2012.09.007

[13] Yang, Y., Pope, S.B., and Chen, J.H. (2013) Empirical low-dimensional manifolds in composition space, Combustion and Flame 160 (10): 1967-1980.

http://dx.doi.org/10.1016/j.combustflame.2013.04.006

[14] Barlow, R.S., Chen, J.-Y., Dally, B., Dreizler, A., Dunn, M.J., Fiorina, B., Geyer, D., Gomez, A., Hochgreb, S., Ihme, M., Janicka, J., Kempf, A., Lindstedt, R.P., Masri, A.R., Meier, W., Pitsch, H., Pope, S.B., Richardson, E.S., Roekaerts, D., Steinberg, A., and Vervisch, L., "Summary", Eleventh International Workshop on Measurement and Computation of Turbulent Nonpremixed Flames (TNF11), 2012.

[15] Barlow, R.S., and Frank, J.H. (1998) Effects of turbulence on species mass fractions in methane/air jet flames, Proceedings of the Combustion Institute 27: 1087-1095. http://dx.doi.org/

[16] Masri, A.R., Kalt, P.A.M., Al-Abdeli, Y.M., and Barlow, R.S. (2007) Turbulence-chemistry interactions in non-premixed swirling flames, Combustion Theory and Modelling 11(5): 653-673. http://dx.doi.org/10.1080/13647830701213482

[17] Pope, S.B. (1985) PDF methods for turbulent reactive flows, Progress in Energy and Combustion

Science 11(2): 119-192. http://dx.doi.org/10.1016/0360-1285

[18] Donde, P., Raman, V., Mueller, M.E., and Pitsch, H. (2013), LES/PDF based modeling of sootturbulence interactions in turbulent flames, Proceedings of the Combustion Institute 34: 11831192. http://dx.doi.org/10.1016/j.proci.2012.07.055,

[19] Haworth, D. (2010) Progress in probability density function methods for turbulent reacting flows, Progress in Energy and Combustion Science 36(2): 168-259.

http://dx.doi.org/10.1016/j.pecs.2009.09.003

[20] Coelho, P. (2012) Turbulence-Radiation Interaction: From Theory to Application in Numerical Simulations, ASME - Journal of Heat Transfer 134 (3): Article Number: 031001. http://dx.doi.org/10.1115/1.4005130 
[21] Merci, B., Roekaerts, D., and Sadiki, A., Experiments and Numerical Simulations of Turbulent Combustion of Diluted Sprays, ERCOFTAC Series Vol. 17, Springer, 2011.

http://dx.doi.org/10.1007/978-94-007-1409-0

[22] McGrattan, K. (2005) Fire modeling: where are we? Where are we going?, Fire Safety Science Proceeding of the $8^{\text {th }}$ International Symposium 8: 53 - 68. http://dx.doi.org/10.3801/IAFSS.FSS.8$\underline{53}$

[23] A. Trouvé and Y. Wang (2010) Large eddy simulation of compartment fires, International Journal of Computational Fluid Dynamics 24 (10): 449 - 466.

http://dx.doi.org/10.1080/10618562.2010.541393

[24] Lautenberger, C.W., de Ris, J.L., Dembsey, N.A., Barnett, J.R., and Baum, H.R. (2005) A simplified model for soot formation and oxidation in CFD simulation of non-premixed hydrocarbon flames, Fire Safety Journal 40 (2): 141 - 176.

http://dx.doi.org/10.1016/j.firesaf.2004.10.002

[25] Wasan, S.R., Rauwoens, P., Vierendeels, J., and Merci, B. (2010), Combustion and Flame 157 (4): 715-734. http://dx.doi.org/10.1016/j.combustflame.2009.12.007

[26] Lautenberger, C., and Fernandez-Pello, C. (2009), Generalized pyrolysis model for combustible solids, Fire Safety Journal 44 (6): 819-839. http://dx.doi.org/10.1016/j.firesaf.2009.03.011

[27] Rein, G., Abecassis-Empis, C., and Carvel, R., The Dalmarnock Fire Tests: Experiments and Modelling, University of Edinburgh, 2007.

[28] Shipp, M., et al. 'Fire spread in car parks', BD2552, Department for Communities and Local Government, December 2010.

[29] Deckers, X., Haga, S., Sette, B., and Merci, B. (2013) Smoke control in case of fire in a large car park: Full-Scale Experiments, Fire Safety Journal 57: 11-21.

http://dx.doi.org/10.1016/j.firesaf.2012.10.017

[30] Beji, T., Verstockt, S., Merci, B., Van de Walle, R., Abecassis-Empis, C., Krajcovic, M., and Majdalani, A., "RABOT2012 - Presentation of the Multi-compartment Full-Scale ('Rabot') Fire Tests", $7^{\text {th }}$ International Seminar on Fire and Explosion Hazards, 2013, pp. 67 - 76. [ISBN: 978981-07-5936-0]

[31] Audouin, L., Chandra L., Consalvi J-L., Gay L., Gorza E., Hohm V., Hostikka S., Ito T., KleinHessling W., Lalleman C., Magnusson T., Noterman N., Park J.S., Peco J., Rigolett L., Suard S., and van Hees P. (2010) Quantifying differences between computational results and measurements in the case of a large-scale well-confined fire scenario, Nuclear Engineering and Design 241(1): 18-31. http://dx.doi.org/10.1016/j.nucengdes.2010.10.027

[32] Rein, G., Torero, J.L., Jahn, W., Stern-Gottfried, J., Ryder, N.L., Desanghere, S., Lázaro, M., Mowrer, F., Coles, A., Joyeux, D., Alvear, D., Capote, J.A., Jowsey, A., Abecassis-Empis, C., and Reszka, P. (2009) Round-Robin Study of a priori Modelling Predictions of The Dalmarnock Fire Test One, Fire Safety Journal 44 (4): 590-602. http://dx.doi.org/10.1016/j.firesaf.2008.12.008

[33] Jahn, W., Rein, G., and Torero, J.L. (2011). Forecasting fire growth using an inverse zone modeling approach, Fire Safety Journal 46 (3): 81-88.

http://dx.doi.org/10.1016/j.firesaf.2010.10.001

[34] Rochoux, M.C., Delmotte, B., Cuenot, B., Ricci, S., and Trouvé, A. (2013) Regional-scale simulations of wildland fire spread informed by real-time flame front observations, Proceedings of the Combustion Institute 34:2641-2647. http://dx.doi.org/10.1016/j.proci.2012.06.090

[35] Cowlard, A., Jahn, W., Abecassis-Empis, C., Rein, G., and Torero, J.L. (2010) Sensor Assisted Fire Fighting, Fire Technology 46: 719-741. http://dx.doi.org/10.1007/s10694-008-0069-1

[36] Davis, W.D., and Forney, G.P., “A sensor-driven fire model version 1.1”, National Institute of Standards and Technology Report NISTIR 6705, Gaithersburg, MD, 2001, 40 p. 
[37] Neviackas, A.W. (2007). Inverse fire modeling to estimate the heat release rate of compartment fires, Master's thesis, University of Maryland. http://hdl.handle.net/1903/7290

[38] Overholt, K.J., and Ezekoye, O.A. (2012) Characterizing heat release rates using an inverse fire modeling technique, Fire Technology 48 (4): 893-909. http://dx.doi.org/10.1007/s10694-011$\underline{0250-9}$

[39] Koo, S.H., Fraser-Mitchell. J., and Welch, S. (2010) Sensor-steered fire simulation, Fire Safety Journal 45 (3): 193-205. http://dx.doi.org/10.1016/j.firesaf.2010.02.003

[40] Beji, T., Verstockt, S., Van de Walle, R., and Merci, B. (2012) On the Use of Real-Time Video to Forecast Fire Growth in Enclosures, Fire Technology (online). http://dx.doi.org/10.1007/s10694$\underline{012-0262-0}$

[41] Verstockt, S., Van Hoecke, S., Beji, T., Merci, B., Gouverneur, B., Cetin, A.E., De Potter, P., and Van de Walle, R. (2013) A multi-modal video analysis approach for car park fire detection, Fire Safety Journal 57: 44-57. http://dx.doi.org/10.1016/j.firesaf.2012.07.005 